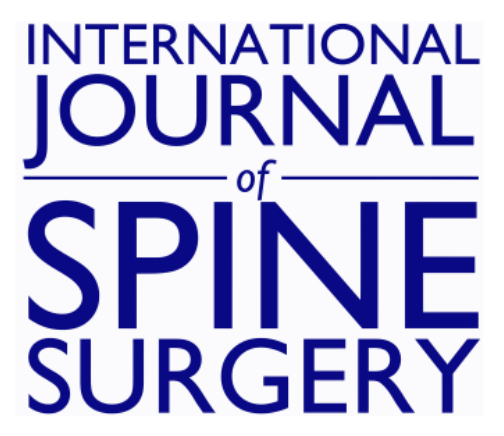

\title{
Cervical Disc Arthroplasty for Axial Neck Pain: Comparison of Outcomes to 2 Other Common Cervical Conditions
}

Matthew F. Gornet, Katrine M. Sorensen and Francine W. Schranck

Int J Spine Surg 2021, 15 (4) 692-700

doi: https://doi.org/10.14444/8091

http://ijssurgery.com/content/15/4/692

This information is current as of April 26, 2023.

Email Alerts Receive free email-alerts when new articles cite this article. Sign up at:

http://ijssurgery.com/alerts

The International Journal of Spine Surgery

2397 Waterbury Circle, Suite 1,

Aurora, IL 60504, Phone: +1-630-375-1432 


\title{
Cervical Disc Arthroplasty for Axial Neck Pain: Comparison of Outcomes to 2 Other Common Cervical Conditions
}

\author{
MATTHEW F. GORNET, MD, ${ }^{1}$ KATRINE M. SORENSEN, MS, ${ }^{2}$ FRANCINE W. SCHRANCK, BSN ${ }^{2}$ \\ ${ }^{l}$ Orthopedic Center of St. Louis, St. Louis, Missouri, ${ }^{2}$ SPIRITT Research, St. Louis, Missouri
}

\begin{abstract}
Background: Cervical disc arthroplasty (CDA) is an established treatment for degenerative disc disease with radiculopathy and/or myelopathy. There is, however, little published evidence of its effectiveness to relieve pain and improve function in patients with a primary diagnosis of axial neck pain. Such patients were excluded from all previous Food and Drug Administration clinical trials for CDA. We compare the outcomes of patients who underwent CDA for 3 common cervical conditions from 2003 to 2018.

Methods: Seven hundred and eighty-two CDA patients at a single site were grouped by primary diagnosis: predominant axial neck pain (ANP) $(n=257)$, predominant radiculopathy (RAD) $(n=331)$, or a combination of both $(A N P+R A D)(n=195)$. Mixed models for repeated measures predicted and analyzed scores at all time points, adjusting for diagnosis group, time point, and, if statistically significant, number of operative levels and demographic characteristics. Outcome measures included the Neck Disability Index, numerical pain scales (intensity plus frequency), the Veterans RAND 12 Item Health Survey (VR-12) Physical Component Score, the Mental Component Score, and reoperations. Patients were assessed preoperatively and postoperatively at 6 weeks, 3 months, 6 months, 1 year, and annually thereafter.

Results: At baseline, arm pain scores in the ANP group were statistically lower $(P=.0002)$ than in the RAD and ANP + RAD groups, consistent with preoperative diagnoses. Surgeries included 40.8\% 1-level, 41.6\% 2-level, 14.7\% 3level, and $2.9 \%$ 4-level. For all outcome measures, improvements were statistically significant from baseline to each postoperative time point without statistical difference between the 3 diagnosis groups. In total, 45/782 patients (5.8\%) underwent a secondary surgery: $3.5 \%$ ANP, $5.8 \%$ RAD, and $8.7 \%$ ANP + RAD. Days to reoperation did not statistically differ between groups $(P=.489)$.

Conclusions: Appropriately selected patients with predominant axial neck pain treated with CDA may achieve clinical and functional outcomes comparable to patients with a primary diagnosis of radiculopathy or of axial neck pain with concomitant radiculopathy.

Clinical Relevance: This study provides information that should help clinicians decide whether to offer CDA for patients with a primary diagnosis of axial neck pain and to appropriately counsel such patients about expected outcomes.
\end{abstract}

Level of Evidence: 4.

Cervical Spine

Keywords: axial neck pain, discogenic pain, radiculopathy, cervical disc arthroplasty, total disc replacement

\section{INTRODUCTION}

Of the 328 conditions studied in the Global Burden of Disease 2016 Study, neck pain was ranked the sixth-highest cause of disability. ${ }^{1}$ From 2006 to 2016, the prevalence of disability caused by neck pain increased by $21.9 \% .{ }^{1}$ Worldwide, nearly half of all individuals will experience an episode of severe neck pain over the course of their lifetimes. ${ }^{2}$ Most episodes of neck pain will resolve within 2 months, but almost $50 \%$ of individuals will continue to have some pain or frequent reoccurrences. ${ }^{3,4} \mathrm{~A}$ 2010 study found that, in their search for treatment, chronic neck pain sufferers had on average 21 visits with more than 5 types of providers. ${ }^{5}$ Similar to back pain, the treatment of neck pain ranges from conservative to surgical care. While the necessity for surgery is evident in cases of neurologic compression, its benefits are not well defined in cases of axial neck pain. ${ }^{6,7}$

Cervical disc arthroplasty (CDA) is now a wellaccepted treatment for radiculopathy and myelopathy, with clinical trial long-term results showing safety and efficacy. However, axial neck pain is a constellation of symptoms without clinically significant radiculopathy or myelopathy, and its surgical 
treatment is still debated. Patients with this diagnosis were excluded from all previous Food and Drug Administration (FDA) clinical trials for CDA. One review was unable to conclude as to the benefits of anterior cervical discectomy and fusion (ACDF) to treat axial neck pain, while others reported significant improvements after ACDF. ${ }^{8,9}$ Separately, the Neck Pain Task Force reporting on the decade 2000-2010 found insufficient evidence to support either fusion or arthroplasty for the treatment of neck pain alone. ${ }^{10}$ More recently, it was reported that preoperative neck pain was a risk factor for persistent neck pain following cervical disc arthroplasty $(\mathrm{CDA}){ }^{11}$

Given the limited evidence regarding the effectiveness of CDA in relieving pain and improving function in patients with a primary complaint of axial neck pain, the purpose of this study is to document the outcomes of patients suffering from predominant axial neck pain due to degenerative disc disease and treated with CDA in clinical practice. These patients are compared to patients with radiculopathy or a combination of axial neck pain and radiculopathy in whom axial neck pain was not the primary complaint in order to assess whether results of CDA for primary axial neck pain are similar to those in patients for whom device clinical trials have previously established safety and efficacy.

\section{MATERIALS AND METHODS}

\section{Patient Sample}

Our research team developed and uses the proprietary SPIRITT database for all spine surgery cases. An approved protocol for assessing preoperative and validated patient-reported outcomes (PROs) is used for all patients. These data are gathered in clinic and remotely via Web-based questionnaires and stored in the database. Data entry codes are used in the database to distinguish primary complaint, with additional complaints and/ or diagnoses coded separately. The measurements used for cervical disc disease in this study are described below. Following approval by a central institutional review board (Western Investigational Review Board, protocol no. 20080163, study no. SR-2013-02), the clinic database was queried to identify all patients who underwent CDA since 2003 with the relevant diagnosis codes. Patients included in this study were treated for 1 of the following diagnoses: predominant axial neck pain, predominant radiculopathy, or a combination of both axial neck pain and radiculopathy. Diagnosis was made by the attending surgeon based on the history and symptoms reported at the time of the patient's office visit and confirmed by physical examination and radiographic studies. The diagnosis of patients with painful cervical pathology can best be depicted as a continuum, with pure radicular symptoms (arm pain, decreased sensation, weakness) at one extreme and pure neck pain at the other. In clinical practice, very few instances of 1 in the complete absence of the other are observed. Radicular arm pain is the most common complaint seen in patients undergoing surgery for cervical degenerative conditions and serves as the starting point for all patient diagnoses. This study classified patients based on their predominant pain complaint, with many patients reporting a relatively equal combination of arm and neck pain symptoms. On preoperative assessment, the group with predominant neck pain had no or dramatically less arm pain than the other 2 groups. Axial neck pain is ill defined in the literature. For this study, axial neck pain is defined as pain contained within the distribution from the occiput to the $\mathrm{C} 7$ spinous process, with or without headaches, that radiates to the trapezius muscle but not to the shoulders and arms, without significant radicular symptoms.

Before surgery, all patients had failed at least 6 weeks of conservative measures, including rest, education, physical therapy or chiropractic care, nonsteroidal anti-inflammatory drugs, and/or injections. Patients with severe radiculopathy unresponsive to initial nonoperative treatment may have gone on to surgery more quickly. For this analysis, the patients were divided into 3 groups: predominant axial neck pain (ANP), predominant radiculopathy (RAD), and axial neck pain with concomitant radiculopathy (ANP + RAD). Those with severe myelopathy, traumatic fracture, or ossification of the posterior longitudinal ligament were excluded because these are different disease processes and generally not considered good candidates for CDA. For the axial neck pain group, operative segments were selected based on high-resolution 3T magnetic resonance imaging with foraminal views. Segments chosen had pathology that correlated with subjective complaints. Structural disc pathology, including annular changes, herniations, degenerations, and osteophytes, were also considered as possibilities for 


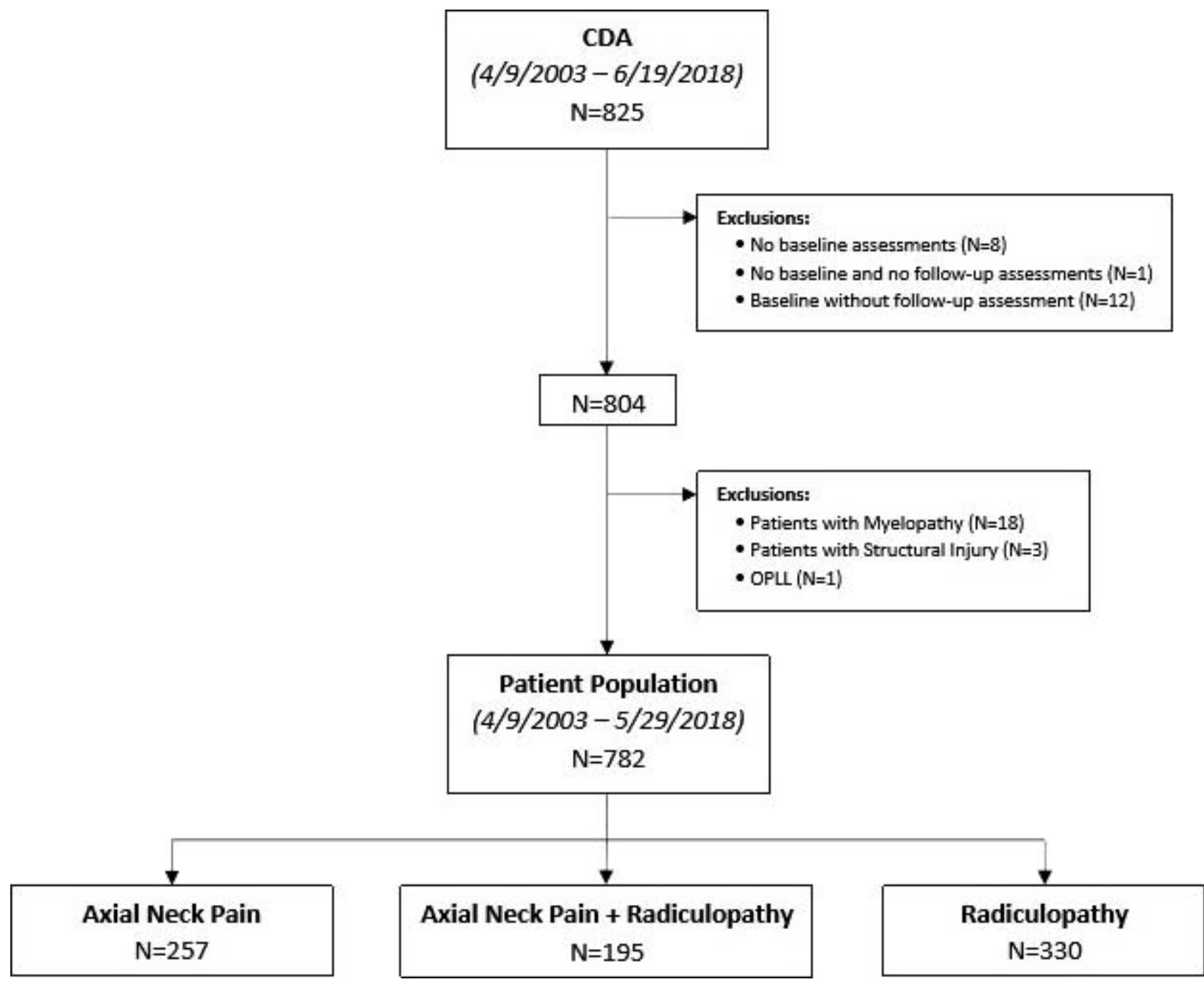

Figure 1. Flowchart of patient inclusion.

the source of neck pain. Severe facet arthropathy was ruled out by computed tomography.

Over the time period used for this study, there was considerable development and evolution of CDA devices, with at least 7 different devices having received FDA marketing approval following clinical trials. Therefore, a variety of different devices were used in this study population as they became available. Choice of device was not related to the diagnoses included here.

From April 2003 to May 2018, 825 patients underwent $\mathrm{CDA}$ at 1 or more levels from $\mathrm{C} 3$ to $\mathrm{C} 7$, with 782 meeting inclusion criteria for this study: 257 for predominant axial neck pain, 331 for predominant radiculopathy, and 195 for combined axial neck pain with radiculopathy (Figure 1). Demographic, surgical, and outcomes data were retrieved from the database for these patients.
Demographic and Surgical Information

Basic demographic information and surgical characteristics were collected: age, sex, body mass index (BMI), race/ethnicity, surgical levels, operative time, turnover time, intravenous fluids, and estimated blood loss.

\section{PROs}

Patients completed validated self-reported measures: Neck Disability Index (NDI), ${ }^{12}$ Veterans RAND 12 Item Health Survey (VR-12) ${ }^{13}$ Physical Component Score (PCS) and Mental Component Score (MCS), and numerical rating scales (intensity + frequency, 0-20 scale) for neck and arm pain. ${ }^{14}$ These measures are those most used in FDA device clinical trials for CDA and are routinely collected on our cervical spine surgery cases. Preoperative and postoperative ( 6 weeks, 3 months, 6 months, 1 
Table 1. Mixed-effects regression models for outcome measures and number of observations used in each model.

\begin{tabular}{lc}
\hline Outcome Measure & No. of Observations \\
\hline NDI & 5147 \\
Neck pain & 5117 \\
Arm pain & 5128 \\
VR-12 PCS & 5007 \\
VR-12 MCS & 5007 \\
\hline
\end{tabular}

Abbreviations: MCS, Mental Component Score; NDI, Neck Disability Index; PCS, Physical Component Score; VR-12, Veterans RAND 12 Item Health Survey.

year, and annually thereafter) scores from these PROs questionnaires were retrieved.

\section{Secondary Surgeries}

The timing, reason, and type of all secondary surgeries to date were recorded.

\section{STATISTICAL METHODS}

Comparisons of demographic characteristics, surgical characteristics, and preoperative PRO scores among the three diagnosis groups were performed using Pearson's chi-square test for categorical data and 1-way analysis of variance with the Student-Newman-Keuls post hoc test for continuous data.

Mixed models for repeated measures (MMRM) statistical analyses were used to assess the effect of diagnosis, time point, and statistically significant covariates (age at surgery, sex, BMI, and number of surgical levels) on each PRO score. MMRM effectively analyze longitudinal data with repeated measures at irregularly timed intervals (eg, weekly, monthly, and yearly) and missing dependent variable data points to produce a score prediction model. Each model then outputs a predicted PRO score for each patient at every time point as long as there are no missing independent variables. Analysis of repeated measures across time using mixed models is common in medical and biological studies and is considered less likely to lead to misinterpretation of results than some of the other methods used to handle missing data. ${ }^{15-17}$ Additionally, these models use all available data, as there is no need to exclude subjects who do not have data at every interval. Therefore, the mixed model was deemed particularly appropriate for this study, which included more than 5000 data points across multiyear intervals for each outcome measure (Table 1). Although the number of patients with postsurgical follow-up differed at any given time interval, the vast amounts of data overall were sufficient to produce score predictions out to 7 years follow-up. Statistical analysis was performed using SAS 9.4 (SAS Institute Inc, Cary, North Carolina).

\section{RESULTS \\ Patient Characteristics (Table 2)}

The sex distribution was statistically different between the three groups: $47 \%$ male in ANP, $60 \%$ male in RAD, and $57 \%$ male in ANP + RAD $(P \leq$ $.007)$, as was mean age (ANP $=43.2 \pm 10.0$ years, $\mathrm{RAD}=45.6 \pm 9.2$, and $\mathrm{ANP}+\mathrm{RAD}=47.4 \pm 9.9 ; P$ $\leq .001)$. In addition, there is a difference in overall distribution of ethnicity between groups $(P \leq .03)$,

Table 2. Patient demographic and surgical characteristics.

\begin{tabular}{|c|c|c|c|c|c|}
\hline & Total $(\mathrm{N}=782)$ & $\operatorname{ANP}(\mathbf{N}=257)$ & $\operatorname{RAD}(\mathbf{N}=330)$ & $\mathbf{A N P}+\operatorname{RAD}(\mathbf{N}=195)$ & $P$ Value $^{\mathrm{a}}$ \\
\hline Male, \% & 55.0 & 47.1 & 59.7 & 57.4 & .007 \\
\hline Age at surgery, mean \pm SD & $45.2 \pm 9.8$ & $43.2 \pm 10.0$ & $45.6 \pm 9.2$ & $47.4 \pm 9.9$ & $<.001^{\mathrm{c}}$ \\
\hline $\mathrm{BMI}$, mean $\pm \mathrm{SD}, \mathrm{kg} / \mathrm{m}^{2}$ & $29.1 \pm 5.8$ & $28.5 \pm 5.9$ & $29.6 \pm 5.8$ & $29.1 \pm 5.5$ & .081 \\
\hline Race/ethnicity, \% & & & & & .030 \\
\hline White or Caucasian & 87.5 & 85.6 & 88.5 & 88.2 & \\
\hline Black or African American & 8.2 & 7.8 & 9.7 & 6.2 & \\
\hline All other ${ }^{\mathrm{b}}$ & 4.4 & 6.7 & 1.8 & 5.6 & \\
\hline Prior decompression, $\%$ & 0.4 & 0.8 & 0.3 & 0.0 & .396 \\
\hline Estimated blood loss, mean $\pm \mathrm{SD}, \mathrm{mL}$ & $53.1 \pm 58.5$ & $50.2 \pm 3.2$ & $57.1 \pm 90.1$ & $50.1 \pm 1.6$ & .269 \\
\hline Intravenous fluids, mean $\pm \mathrm{SD}, \mathrm{mL}$ & $1564.3 \pm 884.8$ & $1616.5 \pm 766.4$ & $1501.2 \pm 1145.5$ & $1601.0 \pm 397.1$ & .245 \\
\hline Operative time, mean $\pm \mathrm{SD}, \min$ & $94.1 \pm 43.2$ & $95.1 \pm 42.1$ & $84.6 \pm 36.1$ & $108.6 \pm 51.0$ & $<.0001^{\mathrm{c}}$ \\
\hline Turnover time, mean $\pm \mathrm{SD}$, min & $63.8 \pm 16.9$ & $65.1 \pm 15.7$ & $63.5 \pm 18.2$ & $62.8 \pm 15.9$ & .429 \\
\hline Number surgical levels, $\%$ & & & & & $<.0001$ \\
\hline 1 & 40.8 & 27.6 & 59.7 & 26.2 & \\
\hline 2 & 41.6 & 45.9 & 34.2 & 48.2 & \\
\hline 3 & 14.7 & 22.2 & 5.8 & 20.0 & \\
\hline 4 & 2.9 & 4.3 & 0.3 & 5.6 & \\
\hline
\end{tabular}

Abbreviations: ANP, axial neck pain; BMI, body mass index; RAD, radiculopathy.

${ }^{\mathrm{a}}$ Boldface values indicate statistical significance.

${ }^{\mathrm{b} I n c l u d e s ~ m i x e d ~ r a c e, ~ H i s p a n i c ~ o r ~ L a t i n o, ~ A s i a n, ~ H a w a i i a n ~ o r ~ P a c i f i c ~ I s l a n d e r, ~ o t h e r, ~ a n d ~ u n k n o w n . ~}$

'Student-Newman-Keuls post hoc test: All groups differed from each other. 
Table 3. Preoperative scores of patient-reported outcomes (mean \pm SD).

\begin{tabular}{|c|c|c|c|c|c|}
\hline & Total $(\mathrm{N}=782)$ & $\operatorname{ANP}(\mathbf{N}=257)$ & $\operatorname{RAD}(\mathbf{N}=330)$ & ANP + RAD $(\mathbf{N}=195)$ & $P$ Value ${ }^{\mathrm{a}}$ \\
\hline NDI score & $53.5 \pm 16.2$ & $\begin{array}{c}55.5 \pm 16.1 \\
\text { VR-12 }\end{array}$ & $52.1 \pm 16.2$ & $53.1 \pm 16.1$ & $.034^{b}$ \\
\hline $\begin{array}{l}\text { Mental Component Score (MCS) } \\
\text { Physical Component Score (PCS) }\end{array}$ & $\begin{array}{l}40.5 \pm 12.0 \\
30.2 \pm 7.8\end{array}$ & $\begin{array}{c}39.5 \pm 11.9 \\
30.3 \pm 8.2 \\
\text { Numeric rating sca }\end{array}$ & $\begin{array}{l}41.4 \pm 12.5 \\
30.1 \pm 7.5\end{array}$ & $\begin{array}{l}40.1 \pm 11.2 \\
30.1 \pm 7.7\end{array}$ & $\begin{array}{l}.164 \\
.960\end{array}$ \\
\hline $\begin{array}{l}\text { Neck pain (intensity }+ \text { frequency) } \\
\text { Neck pain (intensity) } \\
\text { Arm pain (intensity }+ \text { frequency) } \\
\text { Arm pain (intensity) }\end{array}$ & $\begin{array}{r}15.1 \pm 3.3 \\
7.0 \pm 1.8 \\
11.4 \pm 5.5 \\
5.4 \pm 2.7\end{array}$ & $\begin{array}{r}15.3 \pm 3.2 \\
7.1 \pm 1.8 \\
10.3 \pm 5.9 \\
4.9 \pm 3.0\end{array}$ & $\begin{array}{r}14.9 \pm 3.4 \\
6.9 \pm 1.9 \\
12.1 \pm 5.0 \\
5.8 \pm 2.5\end{array}$ & $\begin{array}{r}14.9 \pm 3.3 \\
6.9 \pm 1.8 \\
11.6 \pm 5.4 \\
5.5 \pm 2.7\end{array}$ & $\begin{array}{r}.308 \\
.217 \\
<.001^{c} \\
<.001^{c}\end{array}$ \\
\hline
\end{tabular}

${ }^{a}$ Boldface values indicate statistical significance.

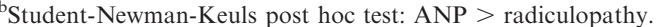

${ }^{\mathrm{c}}$ Student-Newman-Keuls post hoc test: ANP $<$ radiculopathy and ANP $<$ ANP + radiculopathy.

most pronounced in the small number of patients in each group who were not White or African American.

Surgeries were performed at up to 4 levels: $40.8 \%$ 1-level, 41.6\% 2-level, 14.7\% 3-level, and 2.9\% 4level. The number of surgical levels was statistically different between groups $(P<.001)$; the majority of surgeries were 1-level in the RAD group $(59.7 \%)$ and multilevel in the other 2 groups. Although CDA at more than 2 levels is generally considered "off label," it is not uncommon in clinical practice, so these patients have been included. ${ }^{18}$ Operative time also differed between the groups, driven primarily by number of operated levels, with the longest mean time for the combined ANP + RAD group.

\section{Preoperative Scores (Table 3)}

Preoperatively, NDI score was statistically different $(P=.034)$ between the groups overall and specifically differing between ANP (mean $=55.5$ ) and RAD (mean $=52.1$ ), with ANP + RAD not differing significantly from either of the other 2 groups $($ mean $=53.1)$.
Mean arm pain scores were, as expected, also statistically different between the 3 diagnosis groups $(P<.001)$, with post hoc testing finding no statistical differences between the RAD and ANP + RAD groups, in both of which arm pain scores were significantly greater than in the ANP group, corroborating the diagnoses based on clinical presentation.

There were no statistically significant differences between diagnosis groups for the remaining preoperative PRO scores: VR-12 PCS, VR-12 MCS, and neck pain intensity/frequency.

\section{MMRM}

For each of the outcome measures, a mixedeffects regression was performed, controlling for significant patient characteristics. That is, age at surgery, sex, BMI, and number of surgical levels were included in the MMRM only if that variable significantly contributed to the prediction of the outcome variable. Figure 2 provides an example of the fit between actual scores and predicted scores for

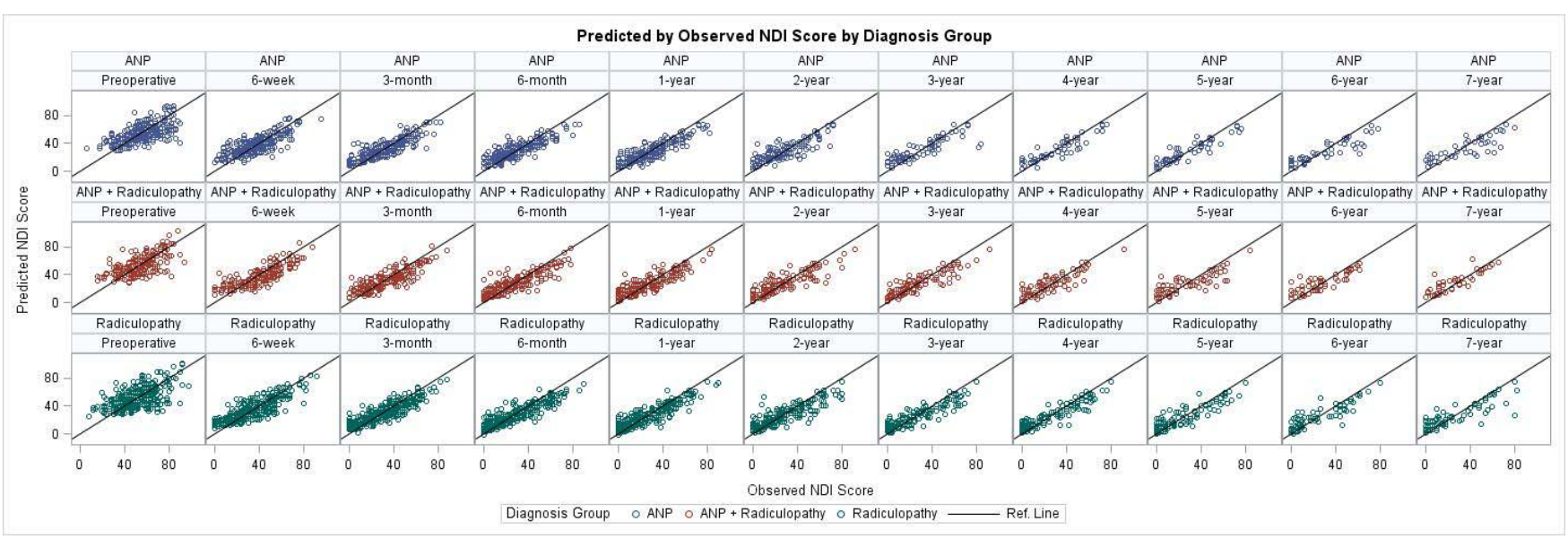

Figure 2. Mixed-effects regression model predicted scores by observed scores for each diagnosis group for the Neck Disability Index as an example. Model shows good fit of predicted scores to observed scores. 
A.

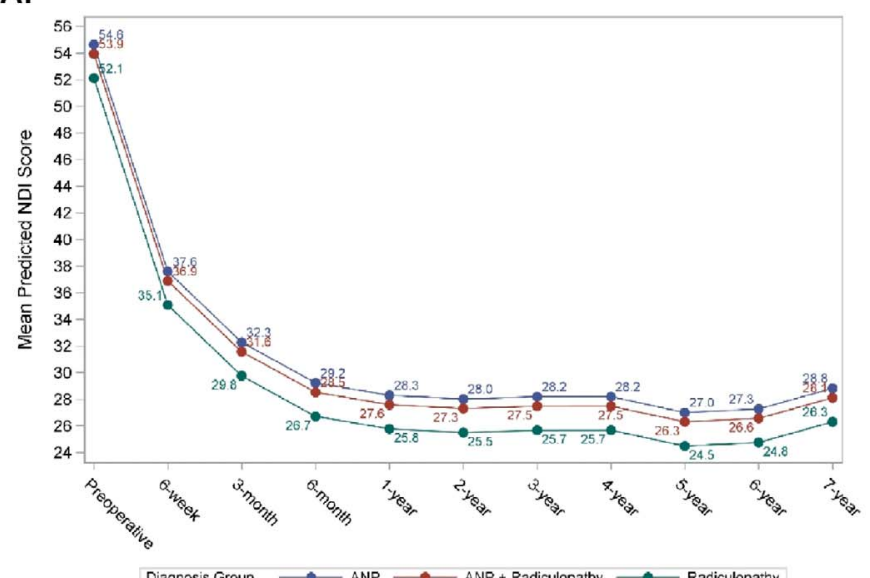

C.

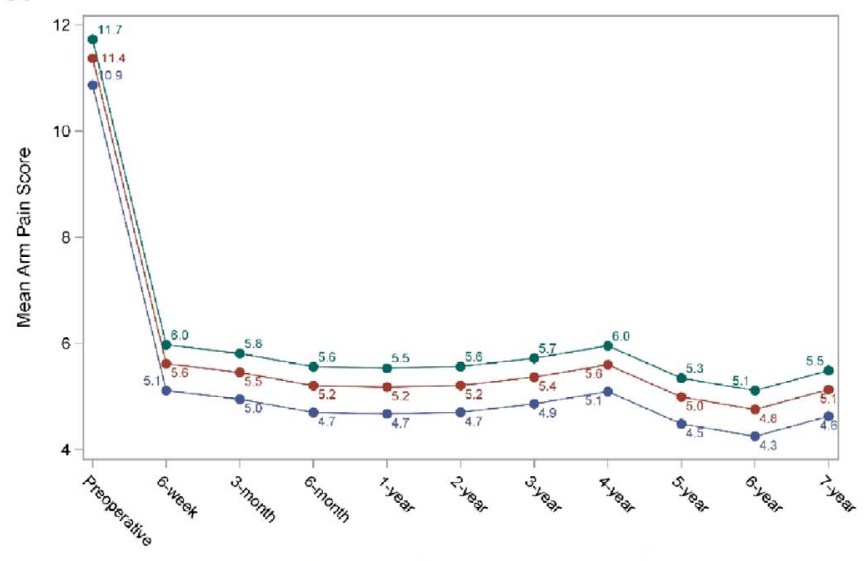

Diagnosis Group $\longrightarrow$ ANP $\longrightarrow$ ANP + Radiculopathy $\longrightarrow$ Radiculopathy

E.

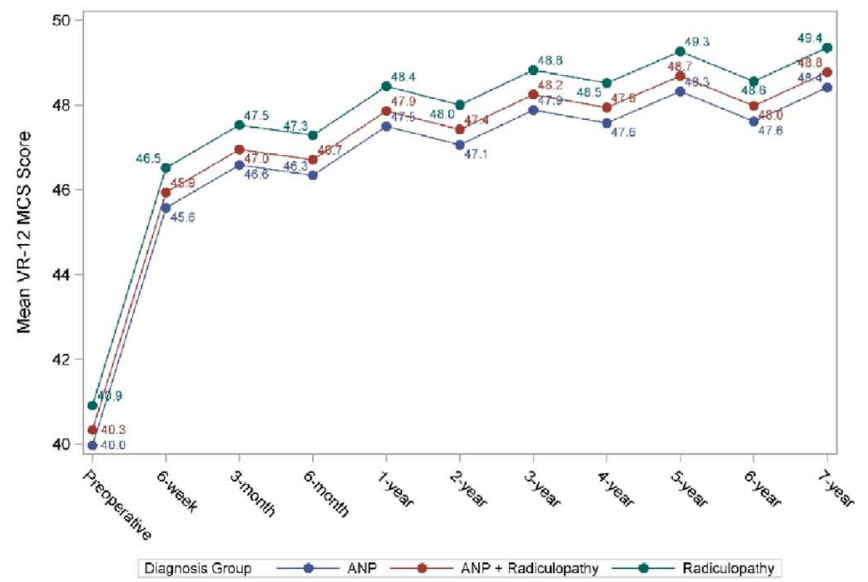

B.

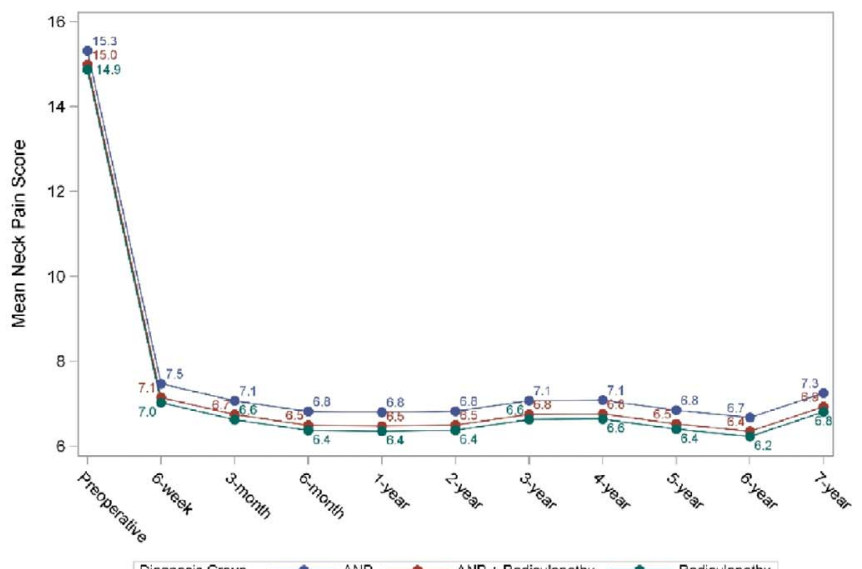

D.

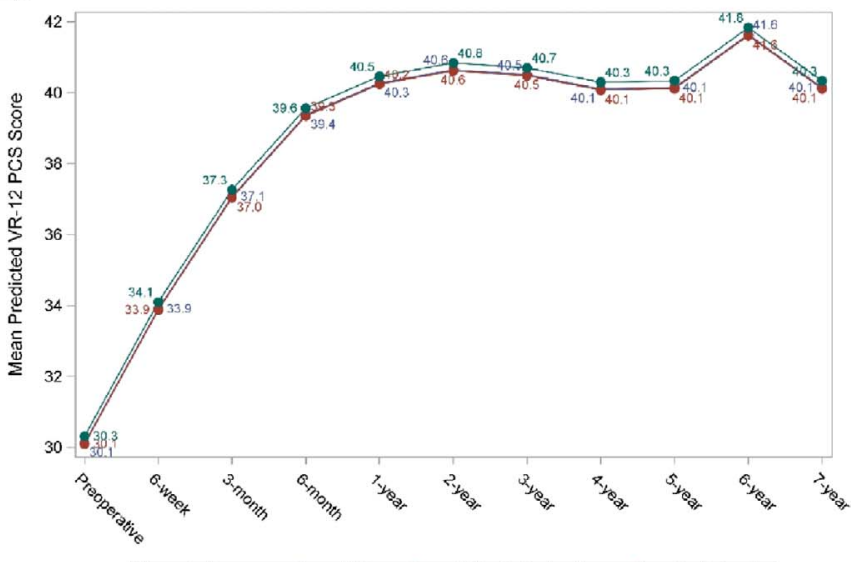

Figure 3. Mean predicted intensity + frequency scores over time by diagnosis group (blue $=$ ANP; red $=$ ANP + radiculopathy; green $=$ radiculopathy). $(A)$ Neck Disability Index. (B) Neck pain (scale 0-20). (C) Arm pain (scale 0-20). (D) Veterans RAND 12 Item Health Survey (VR-12) Physical Component Score. (E) VR-12 Mental Component Score. Time intervals include preoperative, 6 weeks, 3 months, 6 months, 1 year, and annual to 7 years.

the NDI outcome regression model. The figure shows a good fit between the predicted and actual observed scores. Figure 3 shows the results of the MMRM for all 5 of the outcome measures, showing the mean predicted scores at each time interval for each group.
NDI Score (Figure 3A)

In addition to intercept, diagnosis group, and time point, which are always included, the NDI score mixed model includes number of surgical levels $(P=$ $.008)$ and BMI $(P=.015)$. As the number of surgical levels increases, so does NDI score; similarly, as 
Table 4. Secondary surgeries (mean \pm SD).

\begin{tabular}{|c|c|c|c|c|c|}
\hline & Total $(\mathrm{N}=\mathbf{7 8 2})$ & $\operatorname{ANP}(\mathbf{N}=257)$ & $\operatorname{RAD}(\mathbf{N}=330)$ & ANP + RAD $(N=195)$ & $P$ Value \\
\hline Secondary surgery, n (\%) & $45(5.8)$ & $9(3.5)$ & $19(5.8)$ & $17(8.7)$ & .062 \\
\hline Days to second surgery, mean $\pm \mathrm{SD}$ & $993.3 \pm 1135.2$ & $605.3 \pm 851.8$ & $1162.0 \pm 1367.2$ & $1010.2 \pm 979.7$ & .489 \\
\hline Secondary surgery level, n ( $\%$ of 45 secondary surgeries) & & & & & .439 \\
\hline Index & $22(48.9)$ & $6(66.7)$ & $9(47.4)$ & $7(41.2)$ & \\
\hline Adjacent & $18(40.0)$ & $3(33.3)$ & $9(47.4)$ & $6(35.3)$ & \\
\hline Index + adjacent & $2(4.4)$ & $0(0.0)$ & $0(0.0)$ & $2(11.8)$ & \\
\hline Nonadjacent & $3(6.7)$ & $0(0.0)$ & $1(5.3)$ & $2(11.8)$ & \\
\hline Third surgery, $\mathrm{n}$ & 4 & 0 & 2 & 2 & .302 \\
\hline
\end{tabular}

BMI increases, so does NDI. The mean predicted NDI score for each diagnosis group at each time point portrays a significant improvement from baseline for all 3 groups over time $(P<.001$ for all time points) after controlling for all other variables in the model. NDI scores did not statistically differ among the 3 diagnosis groups after controlling for all other variables in the model $(P=.606)$. At 6 weeks postoperative, a greater than 15 -point improvement in mean NDI score is shown for all 3 diagnosis groups, with continued score improvement at each time point until a slight increase at 7 years postoperative.

\section{Neck Pain (Figure 3B)}

The mixed-effects model for neck pain includes intercept, diagnosis group, time point, and sex $(P=$ .015), favoring females. Mean predicted neck pain scores were statistically lower at each follow-up time point compared to baseline $(P<.001$ for all time points) after controlling for all other variables in the model. There were no statistical differences between diagnosis groups $(P<.17)$. A dramatic initial dropoff of greater than $50 \%$ in neck pain scores is seen from baseline to 6 weeks postoperatively, and neck pain scores continued to remain low over time.

\section{Arm Pain (Figure 3C)}

In addition to intercept, diagnosis group, and time point, age at surgery $(P<.001)$ and BMI $(P=.003)$ were also included in the mixed model for arm pain. After controlling for all other variables, mean predicted arm pain is statistically different from baseline to follow-up ( $P<.001$ for all time points), demonstrating significant postoperative improvement. Predicted arm pain scores are not statistically different between the 3 diagnosis groups after controlling for all other variables in the model $(P$ $=.127$ ). Figure $3 \mathrm{C}$ shows a large drop-off in mean postoperative arm pain from baseline to 6 weeks postoperative, and predicted scores remain low out to 7 years.

\section{VR-12 PCS (Figure 3D)}

In addition to intercept, diagnosis group, and time point, the VR-12 PCS mixed-effects model includes age at surgery $(P<.001)$, sex $(P=.013)$, and BMI $(P<.001)$. That is, as BMI and age at surgery increase, the PCS decreases, while PCS scores are predicted to be higher for females than for males. After controlling for all other variables in the model, predicted PCS is statistically improved from baseline to follow-up at each time point $(P<.001$ for all time points) and not statistically different between the three diagnosis groups $(P=.349)$. The mean predicted PCS portrays a steady improvement over time for all diagnosis groups but with a decline at 72 months postoperative.

\section{VR-12 MCS (Figure 3E)}

The model for VR-12 MCS adjusts for the intercept, diagnosis group, and time point only. Number of surgical levels, age at surgery, BMI, and sex were tested, but none of these factors contributed significantly to the prediction of MCS. In this model, MCS is statistically improved from baseline to follow-up for each time point $(P<.001$ for all time points) but not statistically different between the three diagnosis groups $(P=.482)$. The predicted means for each diagnosis group show an initial dramatic predicted MCS improvement from baseline to 6 weeks postoperative and with incremental improvement seen out to 7 years after surgery.

\section{Secondary Surgeries (Table 4)}

A total of 45 patients $(5.8 \%)$ underwent a secondary surgery. Of those, $11(24.4 \%)$ experienced a new injury or trauma, unrelated to their initial symptoms or index treatment, that led to their additional surgery. The proportion of patients undergoing secondary surgeries was not statistically different between the 3 groups, although there was a trend $(P=.06)$ toward a slightly higher rate in the ANP + RAD group at $8.7 \%$. The most frequent 
types of secondary surgery were revision surgery at the index level $(48.9 \%)$ and surgery at the adjacent segment $(40.0 \%)$. Of these 45 patients, $4(8.9 \%)$ also underwent a third cervical surgery ( 2 RAD and 2 ANP + RAD).

The average time elapsed between the index surgery and the second surgery was $1.7 \pm 2.3$ years for ANP, $3.2 \pm 3.7$ years for RAD, and $2.8 \pm 2.7$ years for ANP + RAD. Secondary surgeries appeared to occur sooner in ANP patients, but the difference in time intervals was not statistically different $(P=.489)$ between the 3 groups. This is possibly due to the small number of patients with secondary surgeries and to large standard deviations.

\section{DISCUSSION}

To date, this is the largest known sample with the longest follow-up period of patients suffering from predominant axial neck pain and treated with CDA. Pain and disability levels decreased by about half in the 3 diagnosis groups without favoring 1 diagnosis group over another. The reduction of symptoms is commensurate with the improvement reported in 2 studies of patients suffering from axial neck pain who were treated with ACDF: in 1, reduction in mean NDI score from 58.8 to 30.7 and in mean neck pain from 8.4 to 3.8 was reported for a sample of 87 patients with an average follow-up of 4.4 years. ${ }^{19}$ In another study, a sample of 38 patients with axial neck pain reported a decrease in mean NDI score from 57.5 to 38.9 and in mean neck pain score from 8.3 to 4.1 at an average of 4.4 years after ACDF. ${ }^{20}$

The improvements in PROs of ANP patients in the current study were not statistically different from the improvements reported by RAD patients. Similar results were found in a study comparing the outcomes of patients with axial neck pain $(\mathrm{n}=41)$ and patients with radiculopathy $(\mathrm{n}=161)$ who underwent ACDF. After an average follow-up of 3.3 years, the axial neck pain patients reported a decrease in mean NDI score from 32.6 to 19.8 and mean neck pain from 6.9 to 2.9 , while the radiculopathy patients reported an NDI decrease from 37.0 to 22.4 and neck pain decrease from 6.8 to $3.3 .^{8}$

To our knowledge, only 1 study has previously investigated the outcomes of CDA for patients with axial neck pain. ${ }^{11}$ In that study, 24.4\% (14/45) of axial neck pain patients reported persistent neck pain (at 3 months or later) after CDA. Compared to patients with radiculopathy and/or myelopathy, axial neck pain patients were 3 times more likely to experience persistent neck pain after CDA. In contrast, neck pain scores in the current study improved nearly $50 \%$ by 6 weeks and remained very low up to 7 years postoperative. The decrease in neck pain scores for ANP patients was not statistically different from the neck pain decrease for RAD and ANP + RAD patients, indicating similarly significant improvement.

The current study is a retrospective analysis of the prospectively collected data of a single treatment group from a single institution. As such, it lacks the strength of prospective, randomized, controlled studies. Other potentially confounding factors that might be related to axial neck pain, such as cervical curvature and disc motility, were not included in the current study, nor were some patient characteristics that could potentially affect PROs, such as precipitating cause of injury and duration of symptoms. Future studies will benefit from consistent criteria for classifying patients as having axial neck pain as the primary complaint. In clinical practice, this is based on the history and symptoms reported at the time of the patient's office visit but confirmed by physical examination and radiographic studies. But rarely is the patient complaint strictly all or none when it comes to neck pain. A consistent and/or more objective approach to defining primary axial neck pain, such as the definition we offer in the "Methods" section, will help in making comparisons across the literature. However, there is a clear need for additional evidence regarding the surgical treatment of axial neck pain, particularly with CDA. For that very reason and despite the nonrandomization, the results of this study provide valuable information about the effectiveness of CDA in relieving pain and improving function in patients with a primary diagnosis of axial neck pain. A major strength of this study is the large number of patients, the largest study of its kind to date, as well as the long-term follow-up using a database of regular assessment with standardized measurement tools. Even though not all subjects had follow-up at every interval, a validated statistical method was used that allows for missing data as well as control of possible confounding factors. This statistical method, MMRM, is particularly well suited to studies with large quantities of data collected over many time intervals, and in the present study, enough data points were available on enough 
subjects at every interval to provide predicted values out to 7 years.

\section{CONCLUSIONS}

The results of this study indicate that appropriately selected patients with predominant axial neck pain treated with CDA may achieve success with clinical and functional outcomes comparable to patients with a primary diagnosis of radiculopathy or of axial neck pain with concomitant radiculopathy. Further studies will provide additional confirmation of the effectiveness of CDA in relieving pain and improving function in patients with a primary diagnosis of axial neck pain.

\section{ACKNOWLEDGMENTS}

The authors thank Karen I. Berliner, PhD, for help in manuscript preparation, content suggestions, and editing.

\section{REFERENCES}

1. GBD 2016 Disease and Injury Incidence and Prevalence Collaborators. Global, regional, and national incidence, prevalence, and years lived with disability for 328 diseases and injuries for 195 countries, 1990-2016: a systematic analysis for the Global Burden of Disease Study 2016. Lancet. 2017;390(10100):1211-1259.

2. Fejer R, Kyvik KO, Hartvigsen J. The prevalence of neck pain in the world population: a systematic critical review of the literature. Eur Spine J. 2006;15:834-848.

3. Vasseljen O, Woodhouse A, Bjorngaard JH, Leivseth L. Natural course of acute neck and low back pain in the general population: The HUNT study. Pain. 2013;154:1237-1244.

4. Vos CJ, Verhagen AP, Passchier J, Koes BW. Clinical course and prognostic factors in acute neck pain: an inception cohort study in general practice. Pain Med. 2008;9:572-580.

5. Goode AP, Freburger J, Carey T. Prevalence, practice patterns, and evidence for chronic neck pain. Arthritis Care Res. 2010;62:1594-1601.

6. Buchowski JM, Kelly MP, Barth BM. Axial neck pain, radiculopathy, and myelopathy: recognition and treatment. Practical Pain Manag. 2011;11:1-4.

7. Clarke MJ, Scheifer TK, Pichelmann MA, Krauss WE. Axial neck pain: A surgeon's perspective. Therapy. 2011;8:763770 .

8. Eck JC, Humphreys SC, Hodges SD, Levi P. A comparison of outcomes of anterior cervical discectomy and fusion in patients with and without radicular symptoms. J Surg Orthop Adv. 2006;15:24-26.

9. Riew KD, Ecker E, Dettori JR. Anterior cervical discectomy and fusion for the management of axial neck pain in the absence of radiculopathy or myelopathy. Evid Based Spine Care J. 2010;1:45-50.

10. Carragee EJ, Hurwitz EL, Cheng I, et al. Treatment of neck pain: injections and surgical interventions: results of the
Bone and Joint Decade 2000-2010 Task Force on Neck Pain and Its Associated Disorders. Spine. 2008;33(suppl 4):S153S169.

11. Wagner SC, Formby PM, Kang DG, et al. Persistent axial neck pain after cervical disc arthroplasty: a radiographic analysis. Spine J. 2016;16:851-856.

12. Vernon H, Mior S. The Neck Disability Index: a study of reliability and validity. J Manipulative Physiol Ther. 1991;14:409-415.

13. Boston University School of Public Health. VR-36, VR12 , and $V R-6 D$. http://www.bu.edu/sph/research/researchlanding-page/vr-36-vr-12-and-vr-6d/. Accessed April 19, 2017.

14. McDowell I, Newell C. Measuring Health: A Guide to Rating Scales and Questionnaires. 2nd ed. New York: Oxford University Press; 1996.

15. Hayat MJ, Hedlin H. Modern statistical modeling approaches for analyzing repeated-measures data. Nursing Res. 2012;61:188-194.

16. Lane P. Handling drop-out in longitudinal clinical trials: a comparison of the LOCF and MMRM approaches. Pharm Stat. 2008;7:93-106.

17. Wolfinger RD. An example of using mixed models and PROC MIXED for longitudinal data. $J$ Biopharm Stat. 1997; 7:481-500

18. Gornet MF, Schranck FW, Sorensen KM, Copay AG. Multi-level cervical disc arthroplasty: long-term outcomes at 3 and 4 levels. Int J Spine Surg. 2020;14(S2):S41-S49. doi:10. $14444 / 7090$

19. Garvey TA, Transfeldt EE, Malcolm JR, Kos P. Outcome of anterior cervical discectomy and fusion as perceived by patients treated for dominant axial-mechanical cervical spine pain. Spine. 2002;27:1887-1895; discussion 95.

20. Palit M, Schofferman J, Goldthwaite N, et al. Anterior discectomy and fusion for the management of neck pain. Spine. 1999;24:2224-2228.

Disclosures: The authors report the following potential or perceived conflicts. M.F.G. reports consulting for Aesculap and Medtronic; stock ownership in Bonovo, International Spine \& Orthopedic Institute, LLC, Nocimed, OuroBoros; royalties from Medtronic and RTI. K.M.S. has no conflicts to disclose. F.W.S. reports stock ownership in Nocimed.

Corresponding Author: Matthew F. Gornet, MD, Orthopedic Center of St. Louis, 14825 North Outer Forty Road, Suite 200, St. Louis, MO 61341. Phone: (314) 324-5482; Fax: (314) 548-6221; Email: mfgspine@gmail.com.

Published 19 August 2021

This manuscript is generously published free of charge by ISASS, the International Society for the Advancement of Spine Surgery. Copyright (c) 2021 ISASS. To see more or order reprints or permissions, see http://ijssurgery.com. 